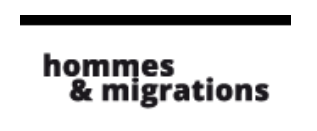

\section{Hommes \& migrations}

Revue française de référence sur les dynamiques

migratoires

$1304 \mid 2013$

Frontières

\title{
La Marche pour l'égalité et contre le racisme, un événement historique ou un coup médiatique?
}

\author{
Mogniss H. Abdallah
}

\section{(2) OpenEdition}

\section{Journals}

Édition électronique

URL : http://journals.openedition.org/hommesmigrations/2681

DOI : 10.4000/hommesmigrations.2681

ISSN : 2262-3353

Éditeur

Musée national de l'histoire de l'immigration

Édition imprimée

Date de publication : 1 octobre 2013

Pagination : 162-166

ISBN : 978-2-919040-24-7

ISSN : $1142-852 X$

\section{Référence électronique}

Mogniss H. Abdallah, «La Marche pour l'égalité et contre le racisme, un événement historique ou un coup médiatique ?», Hommes \& migrations [En ligne], 1304 | 2013, mis en ligne le 24 mars 2014 consulté le 22 septembre 2020. URL : http://journals.openedition.org/hommesmigrations/2681 ; DOI : https://doi.org/10.4000/hommesmigrations.2681 


\section{MÉMOIRES}

\section{LA MARCHE POUR L'ÉGALITÉ ET CONTRE LE RACISME UN ÉVÉNEMENT HISTORIQUE OU UN COUP MÉDIATIQUE ?}

MOGNISS H. ABDALLAH, directeur de l'agence IM'média.

$\mathrm{O}$ ctobre 2013, trente ans après, à la question "Avez-vous déjà entendu parler de la Marche pour l'égalité de 1983, dite Marche des Beurs ?", $81 \%$ des personnes sondées répondent "non". Parmi les 18-24 ans, le "non" grimpe même jusqu'à $90 \%$. Certes, parmi les 65 ans et plus, $27 \%$ disent en avoir entendu parler, surtout en région île-deFrance'. Mais ce sondage ne s'attarde pas sur les confusions récurrentes parmi les réponses positives qui, au vu de nombre d'observations empiriques par ailleurs, amalgament le plus souvent la Marche avec l'opération ultérieure "Touche pas à mon pote" de SOS Racisme². Sans préjuger des effets de réactivation mémorielle ou de relance de la recherche historique suite aux multiples publications, commémorations, réunions publiques ou colloques organisés en 2013, ainsi que de l'impact du film La Marche réalisé par Nabil Ben Yadir sorti le 27 novembre sur les écrans à destination du grand public, ces chiffres concordent avec l'impression dominante : vécue comme un "moment fondateur" d'une France qui se découvre pluriculturelle et pluri-ethnique, voire comme un "événement historique", la Marche n'est pas inscrite pour autant dans le marbre de la mémoire collective. Elle n'est "pas assez entrée dans l'histoire". Mais, à qui la faute ? Poser cette question, c'est aussi une façon d'interroger la notion même d'historicité, c'est-à-dire "la capacité qu'ont les acteurs d'une société ou d'une communauté donnée à inscrire leur présent dans une histoire, à le penser comme situé dans un temps non pas neutre mais signifiant, par la conception qu'ils s'en font, les interprétations qu'ils s'en donnent et les récits qu'ils forgent ${ }^{3}$.

\section{La Marche, un moment fondateur pour certains, un non-événement pour d'autres}

Lors d'un colloque universitaire consacré à "I'héritage de la Marche" organisé les 12 et 13 novembre 2013 à Metz, le sociologue Alain Battegay, qui a lui-même suivi avec empathie l'effervescence sur la place lyonnaise du début des années 1980, émet une hypothèse iconoclaste : "La Marche, dont on sait qu'elle est célébrée par certains comme un événement fondateur a aussi été un non-événement pour d'autres. Je voudrais ici soutenir que cette perception de la Marche comme non-événement est elle-même mémorable, et qu'au lieu de tenir pour acquis le caractère mémorable de la Marche, il convient aussi de documenter et d'analyser les perceptions différenciées de la Marche dans différents cercles, courants d'opinion, milieux professionnels, milieux de l'action publique, de documenter et d'analyser ses ondes et 
ses limites de pertinence à différentes échelles territoriales, sociales, institutionnelles." Alors même que la Marche est représentée comme un temps fort propice à la promotion d'une nouvelle politique de la Ville, Alain Battegay souligne que "les corps d'État impliqués dans les décisions d'aménagement urbain (dont les ingénieurs des Ponts) ont peu été interpellés". Et il ajoute: "Pour une partie importante du monde politique, la Marche a été perçue comme un non-événement, un événement qui ne faisait pas sens", au regard de la société française considérée dans son ensemble. Les réponses évasives de plusieurs artistes ou intellectuels au journal Libération, leur demandant s'ils vont rejoindre le rassemblement final du 3 décembre 1983 à Paris, illustrent cette tendance. "Pardon ? C'est quoi ? dit Philippe Sollers, écrivain. Je suis désolé, je ne suis pas au courant. Je vous lirai demain." "Ils arrivent quand à Paris? Samedi ? demande Françoise Giroud, journaliste. Je ne sais pas si j'irai." "Participer à la Marche samedi ? Pourquoi pas, maintenant que vous me le dites...", songe Edgar Morin, sociologue. Bien que d'autres têtes d'affiche, parmi lesquelles Bernard Henry-Lévy, aient déclaré leur volonté de se joindre "absolument" au défilé 4 , on est bien loin de l'idée reçue d'un enthousiasme débordant et de la mobilisation générale des intellectuels et du Tout-Paris, entretenue par une vision quelque peu mythologique du succès final de la Marche. 


\section{MÉMOIRES}

\section{Des médias moins unanimes et plus ambivalents qu'on ne le dit}

La presse écrite et audiovisuelle a consacré sa rubrique "événement" au "coup de coeur pour les Beurs" (Le Matin, 5 décembre 1983). Mais l'accueil de la Marche par les médias ne fut pas unanime, sans anicroches. Certaines rédactions se révèlent clivées. "Paris vaut bien une marche... mais les contrôles d'identité et les rafles se poursuivent", titre Le Quotidien de Paris du 3 décembre, comme pour mieux insinuer que l'idylle entre les marcheurs et l'État socialiste est un leurre, une simple posture "antiraciste". Le Figaro publie le 5 décembre 1983 une tribune du révérend père Riquet sobrement intitulée "Raison garder" : "Tous les chrétiens devraient être d'accord avec la Bible" écrit-il, "pour dire quand un immigrant s'installera chez vous, dans votre pays (...) vous le traiterez comme un autochtone, comme l'un de vous, vous l'aimerez comme vous-mêmes

Dès 1982, une étude remarquée

sur l'insertion des jeunes d'origine étrangère alerte sur "le risque de graves difficultés sociales" si le pays laisse en marge cette fraction de la population en passe de devenir majoritaire dans l'immigration.

(...). Il ne s'ensuit pas que le gouvernement de la France doive demain être confié à des hommes dont la culture et les moeurs seraient profondément différentes des nôtres." Le 13 décembre, France-Soir tacle la réception chez le président Mitterrand de Toumi Djaïdja, figure emblématique de la Marche : "Le 'marcheur' reçu à l'Élysée avait fait l'objet d'un mandat d'amener", fac-similé à l'appui. Le procès en légitimité des "gentils Beurs" est déjà enclenché.
Pour évaluer l'impact public positif de la Marche, d'aucuns limitent leur horizon médiatique à la presse réputée "pro-immigrée" (Libération, Le Monde, Le Matin, Le Nouvel Obs, Actuel, etc.) et à quelques journaux télévisés ou émissions, tels le magazine hebdomadaire Résistances de Bernard Langlois sur Antenne 2 ou encore Mosaïque, l'émission destinée aux communautés immigrées sur FR3. L'historien Gérard Noiriel prend comme chose acquise l'antiracisme du journal Libération, à l'origine selon lui de l'appellation "Marche des Beurs". "La thématique antiraciste, nourrie de références américaines (Martin Luther King), était déjà 'routinisée' comme un type d'événement dont il fallait tenir compte", affirme-t-il|s. C'est méconnaître les conditions même de la production de la couverture de la Marche par le journal, faire fi des motivations personnelles et des engagements différenciés des journalistes impliqués. Durant l'été 1983, bien que l'idée de la Marche ait été déjà discutée lors de rencontres publiques dans la banlieue lyonnaise, Libération pourtant présent ne la relaie pas encore. Son correspondant local, Robert Marmoz, mentionne en revanche "les mères des victimes de crimes racistes" qui, à l'instar des "folles de mai argentines", "défileront à la rentrée dans les rues de Paris avec les portraits de leurs enfants pour lesquels elles réclament justice ${ }^{6 "}$. Et lorsque Mohamed Nemmiche, un jeune du journal interculturel immigré Sans Frontière, pigiste occasionnel de la rubrique "Tête d'affiche" de Libération, vient proposer début octobre 1983 un carnet de route quotidien de la Marche, il se fait rembarrer par Serge July. Au départ de la Marche, ce sera un pool de médias dédiés et de radios libres, ainsi que des journalistes ou photographes indépendants, qui assureront la couverture médiatique initiale. 
En ce début des années 1980, dans les rédactions les plus "branchées", un climat de défiance visà-vis de l'antiracisme rejaillit de temps à autre : "Vous êtes de vieux gauchistes, des professionnels de l'antiracisme. C'est votre raison sociale. S'il n'y a plus de racisme, que devenez-vous ? Vous avez donc intérêt à en voir partout." Voilà comment, en mai 1981, Yannick Blanc, rédacteur vedette du magazine Actuel, polémique avec des jeunes de la cité Bassens à Marseille. "Vous voulez des journalistes lèche-cul qui répètent à longueur d'articles: 'Pauvres, pauvres, pauvres Arabes.' Vous pensez que vous allez me culpabiliser avec votre chantage au racisme? Quelle rigolade! Dans une génération, les Arabo-Français auront leurs businessmen, leurs bourgeois, leurs artistes", s'énerve-t-il dans un "droit de réponse"7. D'Actuel à Libération, on retrouvera cette ode à l'élite franco-arabe, comme dans l'éditorial de Serge July intitulé "Le pari des Beurs", publié au lendemain de l'arrivée triomphale de la Marche à Paris. Libération a senti la montée en puissance de la mobilisation du gouvernement en faveur des "Beurs" et décide alors de l'accompagner, ce qui, en creux, met en évidence l'interdépendance entre médias et pouvoirs publics.

\section{Se recentrer sur les jeunes, une injonction institutionnelle}

On présente souvent l'accès des "Beurs" à l'espace public, et en particulier la "mode beur", comme une conséquence directe de la Marche. Il est indéniable qu'il y a eu un phénomène d'amplification, une visibilité inédite. Mais l'incitation initiale est antérieure et correspond à des préoccupations institutionnelles, et à la nécessité de relancer l'esprit d'expérimentations novatrices dans l'industrie culturelle apparu après la victoire de la gauche en 1981 qui montre déjà des signes d'essoufflement. Dès 1982, une étude remarquée sur l'insertion des jeunes d'origine étrangère alerte sur "le risque de graves difficultés sociales" si le pays laisse en marge cette fraction de la population en passe de devenir majoritaire dans l'immigration ${ }^{8}$. Une autre étude sur "l'information et l'ex- Les jeunes marcheurs pression culturelle des ont sans doute été dépassés communautés immigrées parleurimage publique en France", dit "rapport et n'avaient pas eux-mêmes Françoise Gaspard", égale- vraiment conscience ment sortie en 1982, consi- de la portée historique de dère les "jeunes de la deu- leur épopée. xième génération" comme des "agents du mélange des cultures" et avance des propositions pour les aider "à se remettre en jeu", "en redéployant à leur avantage les subventions du Fonds d'action sociale (FAS)". II s'agit alors de favoriser "les formes culturelles nouvelles" dans les médias, la musique, et "l'accès aux modes de création audiovisuelle pour faire partager le regard que les jeunes portent sur eux-mêmes". Ces "propositions", formulées par de hauts fonctionnaires d'État, prennent des allures d'injonction institutionnelle à se recentrer sur les jeunes : l'émission Mosaïque sur FR3, Sans Frontière et nombre d'associations sont incitées à mettre en œuvre cette politique.

La cure de jouvence imposée d'en haut suscite néanmoins de fortes réticences, qui s'exprimeront ouvertement au lendemain de la Marche. La grande exposition Les Enfants de l'immigration qui se tient au Centre Georges-Pompidou (Beaubourg) de janvier à avril 1984 donne à voir de multiples petits espaces d'expression sociale ou culturelle des jeunes, puis se clôt par un imposant espace intitulé "Construction", celui des pers- 


\section{MÉMOIRES}

pectives, où “les 'aînés' peuvent jouer un rôle d'entraînement, d'identification". Les "aînés", ceux qui savent, enseignants, universitaires, militants politisés aussi, reprennent la main par rapport à des jeunes trop imprévisibles. Une partie des animateurs de Convergence 84, la deuxième Marche à mobylette, dénoncera ce "fraternalisme" qui avait amené en son temps Aimé Césaire à écrire le 24 octobre 1956 une retentissante lettre de démission du PCF.

\section{Télescopage entre mémoire et histoire}

Les jeunes marcheurs ont sans doute été dépassés par leur image publique et n'avaient pas euxmêmes vraiment conscience de la portée historique de leur épopée. Du moins, pas encore. Tels des "historiens de l'instant", Bouzid Kara a laissé une trace avec son livre La Marche, traversée de la France profonde (éditions Sindbad, 1984, réédité en novembre 2013 par Actes Sud), et Farid L'Haoua a légué à la postérité une couverture photographique plutôt exhaustive de l'événement vu du point de vue des marcheurs. Au moment de la sortie de son livre, Bouzid Kara expliqua qu'il l'avait écrit avant tout en hommage à ses compagnons de route. II circulera pourtant bien audelà des cercles d'initiés. Un exemple parmi d'autres : un extrait en est publié sur une double page d'un manuel scolaire de français pour les élèves des lycées d'enseignement professionnel chez Nathan en septembre 1985. Mais, au-delà du volontarisme de quelques-uns, le point de vue des acteurs est en général réduit à une "microhistoire". Trente ans après, la frénésie commémorielle consécutive à une nouvelle commande publique ${ }^{10}$ va faire sortir les "vrais" marcheurs de leur léthargie. Par sursaut d'orgueil, ces derniers n'entendent pas une fois de plus se faire déposséder de leur propre expérience. Le film La Marche, de Nabil Ben Yadir, les stimule. Ils se montrent plutôt indulgents vis-à-vis du film parce qu'il les met en valeur, tels des héros retrouvés. Mais comme c'est une fiction cinématographique, ils ont décidé de raconter "la véritable histoire qui a inspiré le film", parfois sous une forme romanesque ${ }^{11}$. Une douzaine d'entre eux fondent au cour de l'année l'Association des marcheurs historiques de 1983. Au risque de cultiver un entresoi peu propice à l'enjeu du nécessaire passage des dimensions privées de la mémoire à une histoire partagée, au-delà donc des seuls acteurs de la Marche et des milieux concernés. La séquence "souvenirs et émotions" ouverte par une commémoration sacralise le témoignage "authentique" des marcheurs, sans s'interroger sur la construction de celui-ci. Elle laisse dès lors peu d'espace pour une réflexion critique, un tant soi peu distanciée, sur les dynamiques complexes de la Marche, sur la consistance réelle ou supposée de son message "pour l'égalité et contre le racisme", et sur sa mise en perspective dans une société marquée par des discriminations raciales et sociales aux formes renouvelées. Néanmoins, des chantiers au long cours sont ébauchés : des bribes d'archives personnelles inédites émergent sur les réseaux sociaux, des travaux d'inventaire et de restauration d'archives écrites, sonores, photographiques ou audiovisuelles se multiplient. Autant de matière première pour les héritiers de la Marche, les chercheurs et le public profane, à même de permettre la production d'une connaissance plus approfondie d'un événement au statut à ce jour encore incertain. 